\title{
Comunicação
}

[Communication]

\section{Sincronização da ovulação utilizando FSH em substituição à eCG em cabras}

\author{
[Ovulation synchronization using FSH replacing eCG in goats] \\ R.S. Souza, ${ }^{1}$, L.P. Barbosa ${ }^{2 *}$, C.S. Aguiar $^{2}$, J. Figueredo Júnior ${ }^{2}$, M.O. Ribeiro ${ }^{2}$, C.S. Mendes ${ }^{2}$, \\ V.F. Almeida ${ }^{2}$, R.C.S.A. Araújo ${ }^{2}$, A.M. Pinheiro ${ }^{2}$, J.A. Marques $^{2}$ \\ ${ }^{1}$ Universidade Federal da Bahia - UFBA - Salvador, BA \\ ${ }^{2}$ Universidade Federal do Recôncavo da Bahia - UFRB - Cruz das Almas, BA
}

\begin{abstract}
A caprinocultura do Nordeste apresenta baixos níveis de produtividade, tanto na produção de carne quanto na de leite (Anuário..., 2006). Dentro deste panorama, torna-se importante a adoção de biotécnicas da reprodução para incrementar a eficiência produtiva dos animais e promover o rápido crescimento na qualidade genética do rebanho.
\end{abstract}

A utilização de hormônio com ação folículo estimulante em protocolos de sincronização da ovulação tem resultado em melhor viabilidade do oócito, com maior taxa de prenhez (Baruselli et al., 2004). A gonadotrofina coriônica equina (eCG) é utilizada como estimuladora da superovulação, contudo tem alto peso molecular e induz à formação de anticorpos anti-eCG, se usada em aplicações repetidas. Uma alternativa ao uso de eCG é a utilização do hormônio folículo estimulante (FSH) que, puro ou associado ao hormônio luteinizante (LH), também estimula a superovulação, apresenta reação imunológica menor, maior disponibilidade e possui aceitação em vários países (Oliveira et al., 2007).

Desta forma, este trabalho teve o objetivo de avaliar a utilização do FSH em substituição à eCG no comportamento estral e na dinâmica folicular de cabras leiteiras submetidas a protocolos de sincronização da ovulação.

Doze fêmeas das raças Parda Alpina e Saanen (mestiças), escolhidas com base na condição corporal e na atividade ovariana, foram submetidas a regime semi-intensivo de produção.
Durante o dia, os animais tiveram livre acesso à pastagem nativa (Brachiaria decumbens) e receberam concentrado à base de farelo de milho e soja. Água e suplemento mineral foram fornecidos à vontade.

Todas as cabras receberam esponjas intravaginais impregnadas com $60 \mathrm{mg}$ de acetato de medroxiprogesterona (MAP; Progespon ${ }^{\circledR}$, Syntex, Argentina) durante 10 dias. No momento da retirada das esponjas, os animais foram distribuídos aleatoriamente em dois gupos (G). Os do $\mathrm{G} 1(\mathrm{n}=6)$ receberam aplicação de $0,5 \mathrm{~mL}$ de um análogo sintético da $\alpha$ PGFð cloprostenol (Prolise ${ }^{\circledR}$, Arsa SRL, Argentina), e 100UI de eCG (Novormon ${ }^{\circledR}$, Syntex, Argentina) para cada $10 \mathrm{~kg}$ do peso vivo, ambos aplicados via intramuscular (IM), e os do G2 (n=6) aplicação de $0,5 \mathrm{~mL}$ de um análogo sintético da PGF $2 \alpha$, o d-cloprostenol (Prolise ${ }^{\circledR}$, Arsa SRL, Argentina), e 20mg de FSH (Folltropin ${ }^{\circledR}$, Bioniche, Canadá) (IM).

Após a retirada das esponjas, os animais foram monitorados para detecção do estro com o auxílio de um rufião. Os sinais de estro observados foram: inquietação, micção frequente, vocalização, procura pelo macho, agitação de cauda, hiperemia e edema vulvar, descarga vaginal de muco cristalino, comportamento homossexual e imobilidade à monta. Considerando-se como início do estro o momento em que começou o interesse do rufião pela fêmea, a recusa em aceitar a monta e a regressão do edema vulvar foram interpretados como fim do estro.

Recebido em 14 de fevereiro de 2011

Aceito em 23 de março de 2011

*Autor para correspondência (corresponding author)

E-mail: lpires73@yahoo.com.br 
Para avaliação da intensidade do estro, foi utilizada uma escala de 0 a 3 , em que 0 corresponde à ausência total de sinais de estro e 3 à intensidade acentuada. $\mathrm{O}$ acompanhamento dos ovários foi iniciado seis horas após a retirada da esponja, em intervalos de seis horas, por ultrassonografia, e finalizado 12 horas após a ovulação. As imagens dos ovários foram obtidas por via transretal utilizando-se ultrassom, marca Pie Medical (modelo Áquila Vet), acoplado a um transdutor linear de 6.0MHz.

A ovulação foi calculada pela média entre a hora da última visão do folículo ovulatório e a hora em que se detectou a ovulação. A taxa de crescimento folicular (TCF) (mm/dia) foi obtida por meio da diferença dos diâmetros final (Df) e inicial (Di), dividida pelo número de horas (h) e multiplicada por $24-\mathrm{TCF}=\mathrm{Di}-\mathrm{Df} / \mathrm{h}$ x $24-\mathrm{a}$ fim de se alcançar a taxa de crescimento diário. As medições dos ovários foram obtidas pelo maior diâmetro deles. O diâmetro folicular foi obtido pelo maior comprimento da cavidade antral a partir de $0,30 \mathrm{~cm}$. O folículo dominante foi o que apresentou o maior diâmetro e que excedeu ao de todos os demais. Considerou-se apenas um folículo subordinado, sendo classificado como um dos que apareceram simultaneamente com o folículo dominante, porém com menor diâmetro e de menor persistência.

Para análise dos dados, primeiro foi verificada a normalidade das variáveis. Algumas delas não apresentaram distribuição normal, o que as indica para testes não paramétricos. Neste caso, utilizou-se o teste Wilcoxon, que é uma variante do teste Kruskal-Wallis, quando se têm apenas dois tratamentos com distribuição das variáveis não normais. Foi realizada análise de variância para as variáveis que apresentaram distribuição normal, e adotou-se o nível de significância de $5 \%$.

Todas as cabras exibiram comportamento estral após a retirada das esponjas (Tab. 1). A totalidade dos animais em estro demonstrou a eficiência dos dois tratamentos de indução de estro, o que, segundo Prosperi et al. (2003), evidencia que a associação entre progestágenos e gonadotrofinas é eficiente na indução do estro em caprinos. Ocorre relação positiva entre a exibição de comportamento estral e bons resultados de taxa de gestação com a utilização de protocolos de sincronização, além da grande importância dessa exibição quando se usa inseminação artificial ou cobertura natural com detecção de estro.

Tabela 1. Comportamento estral de cabras leiteiras submetidas a protocolos utilizando FSH em substituição à eCG

\begin{tabular}{lccc}
\hline \multicolumn{1}{c}{ Parâmetro } & G1 (eCG) & G2 (FSH) & Total \\
\hline AE (\%) & $100(6 / 6)$ & $100(6 / 6)$ & $100(12 / 12)$ \\
IRIE (h) & $45 \pm 3,5$ & $38,6 \pm 2,12$ & $41,2 \pm 7,9$ \\
IRFE (h) & $70,9 \pm 3,2$ & $69,6 \pm 2,9$ & $70 \pm 2,9$ \\
DE (h) & $25,5 \pm 0,5$ & $31 \pm 6,8$ & $28,8 \pm 5,8$
\end{tabular}

$\mathrm{AE}=$ animais em estro; IRIE=intervalo entre a retirada da esponja e o início do estro; IRFE=intervalo entre a retirada da esponja e o final do estro; $\mathrm{DE}=$ duração do estro. Não houve diferença significativa entre os grupos $(\mathrm{P}>0,05)$.

Segundo Rubianes et al. (1996), a fertilidade é reduzida quando o intervalo da retirada do dispositivo - início do estro - é maior que 30 horas, em razão da presença de anticorpos antieCG, que atrasam a onda pré-ovulatória de LH e o momento de ovulação. O intervalo da retirada da esponja - final do estro - está diretamente relacionado com a duração do estro e pode ter sido influenciado pelo procedimento de detecção do estro.

Os parâmetros de dinâmica folicular não foram influenciados pelos protocolos $(\mathrm{P}>0,05)$. (Tab. 2). 
Sincronização da ovulação...

Tabela 2. Dinâmica folicular de cabras leiteiras submetidas a protocolos de sincronização utilizando FSH em substituição à eCG

\begin{tabular}{lccc}
\multicolumn{1}{c}{ Parâmetro } & G1 (eCG) & G2 (FSH) & Total \\
\hline TOE (cm) & $1,60 \pm 0,15$ & $1,78 \pm 0,18$ & $1,64 \pm 0,18$ \\
TOD (cm) & $1,48 \pm 0,25$ & $1,70 \pm 0,12$ & $1,57 \pm 0,23$ \\
TMF (cm) & $0,93 \pm 0,11$ & $0,94 \pm 0,14$ & $0,94 \pm 0,11$ \\
TSMF (cm) & $0,73 \pm 0,09$ & $0,76 \pm 0,05$ & $0,74 \pm 0,07$ \\
IREO (h) & $78,87 \pm 14,6$ & $84,33 \pm 6$ & $81,60 \pm 10,29$ \\
IIEO (h) & $48,23 \pm 4,68$ & $39,33 \pm 3,18$ & $43,78 \pm 4,71$ \\
NO & $1,5 \pm 0,5$ & $1,5 \pm 0,5$ & $1,5 \pm 0,5$ \\
TCF (mm/d) & $1,35 \pm 0,53$ & $0,85 \pm 0,54$ & $0,88 \pm 0,51$
\end{tabular}

TOE=tamanho do ovário esquerdo; TOD=tamanho do ovário direito; TMF=tamanho do maior folículo; TSMF=tamanho do segundo maior folículo; IREO= intervalo entre a retirada da esponja e a ovulação; IIEO=intervalo entre o início do estro e a ovulação; $\mathrm{NO}=$ número de ovulações. TCF=taxa de crescimento folicular. Não houve diferença significativa entre os grupos $(\mathrm{P}>0,05)$.

Protocolos de longa duração resultam em concentrações subluteais de progesterona, que, por sua vez, podem promover excessivo crescimento e persistência de grandes folículos dominantes, resultando em ovulação de má qualidade, em decorrência da redução da viabilidade do oócito (Cruz et al., 2006). Isto pode ter acontecido no presente estudo. O tamanho do segundo maior folículo, $\mathrm{G} 1=0,73 \mathrm{~cm}$ e $\mathrm{G} 2=0,76 \mathrm{~cm}$, não diferiu entre grupos $(\mathrm{P}>0,05)$ quanto ao diâmetro máximo alcançado.

O atraso no intervalo entre o início do estro e a ovulação pode ser consequência da formação de anticorpos anti-eCG, segundo Leite et al. (2006). De acordo com Roy et al. (1999), os anti-eCG atrasam o pico pré-ovulatório de LH e o momento da ovulação.

O número médio de ovulações nos dois protocolos não diferiu entre os grupos e está dentro da média da espécie. Também a taxa de crescimento folicular foi semelhante entre os grupos. Leyva et al. (1998) sugeriram que a taxa de crescimento folicular em alguns folículos, quando se compara tal crescimento com outros que crescem mais rapidamente, pode desencadear fortes implicações na qualidade do oócito no momento da ovulação.

A utilização da eCG pode afetar os mecanismos responsáveis pelo crescimento folicular e aumentar a taxa ovulatória, pela redução no diâmetro de folículos menores, ou pelo recrutamento de folículos de menor diâmetro (Driancourt et al., 1993). Esta condição pode estar relacionada à sua vida média longa que pode ter efeitos negativos na sequência normal dos eventos fisiológicos do desenvolvimento folicular e maturação oocitária (Donrov et al., 1998).

O uso do FSH pode ser efetivo para a indução de ovulação e fornecer embriões viáveis para a criopreservação e subsequente transferência. Porém, se utilizado de forma inadequada, pode promover alguns problemas, tais como falha na ovulação dos folículos, uma vez atingido o tamanho pré-ovulatório e estimulação exagerada do ovário, acompanhada por uma pobre recuperação embrionária por ovulação (Squires, 2005).

Desse modo, concluiu-se que o estro e a ovulação podem ser eficientemente induzidos e sincronizados em cabras por meio de exposição ao progestágeno, associado à administração de eCG ou FSH, e que a escolha de um ou outro protocolo deve estar condicionada ao preço de aquisição dos hormônios ou de outros fatores que não a dinâmica folicular ovariana e o comportamento estral.

Palavras-chave: progestágenos, dinâmica folicular, ultrassonografia 


\begin{abstract}
The effect of substitution of equine chorionic gonadotropin (eCG) by follicle stimulating hormone (FSH) in synchronization protocols of ovulation in dairy goats was evaluated. Twelve goats received intravaginal sponges impregnated with $60 \mathrm{mg}$ of medroxyprogesterone acetate (MAP) for 10 days. The sponges were removed and the animals were distributed into two groups $(G)$ : $G 1(n=6)$ treated with $0.5 \mathrm{~mL}$ of a synthetic analogue of PGF2 $\alpha$ and $100 \mathrm{IU}$ of eCG for each $10 \mathrm{~kg}$ weight, intramuscular injection (IM); and G2 (n=6) treated with $0.5 \mathrm{~mL}$ of a synthetic analogue of PGF2 $\alpha$ and $20 \mathrm{mg}$ of FSH (IM). All animals were monitored for estrus detection with aid of a ruffian after sponge removal. The ovarian dynamics were analyzed by ultrasound, since six hours after sponge removed. Each animal was analyzed in time elapsed of six hours until 12 hours after ovulation detection. For data analyses, the Wilcoxon test and variance analyses were used. There was not difference between the analyzed parameters $(P>0.05)$. In this way, eCG can be replaced by FSH in synchronization protocols of ovulation in dairy goats.
\end{abstract}

Keywords: progestogens, folicular dynamics, ultrasonography

\section{REFERÊNCIAS BIBLIOGRÁFICAS}

ANUÁRIO DA PECUÁRIA BRASILEIRA. São Paulo: Instituto FNP, 2006.

BARUSELLI, P.S.; MADUREIRA, E.H.; MARQUES, M.O. et al. Efeito do tratamento com eCG na taxa de concepção de vacas Nelore com diferentes escores de condição corporal inseminadas em tempo fixo. Acta Sci. Vet., v.32, p.228, 2004.

CRUZ, J.F.; QUEIROZ JÚNIOR, P.; BONOMO, P. et al. O uso do benzoato de estradiol em protocolo de indução de estro em cabras leiteiras durante anestro estacional. In: REUNIÃO ANUAL DA SOCIEDADE BRASILEIRA PARA O PROGRESSO DA CIÊNCIA, 58., 2006, Florianópolis. Anais... Florianópolis, SC: SBPC, 2006.

DONROV, T.S.; BATSAIHAN, D.; LEY, W.B. Gonadotrophin extraction from pregnant mare's serum and effect of PMSG preparation on the fertility of Mongolian native ewes. Small Ruminant Res., v.28, p.61-66, 1998.

DRIANCOURT, M.A.; GOUGEON, A.; ROYÈRE, D. Ovarian function. In: THIBAULT, C.; LEVASSEUR, M.C. (Eds.) Reproduction in mammals and man. Amsterdam: Elsevier, 1993. p.283-305.

LEITE, P.A.G.; CARVALHO, G.R.;. RODRIGUES, M.T. et al. Indução da ovulação em cabras, fora da estação reprodutiva, com LH e GnRH e com estro induzido por progestágenos. Arq. Bras. Med. Vet. Zootec., v.58, p.360-366, 2006.
LEYVA, V.; BUCKRELL, B.C.; WALTON, J.S. Regulation of follicular and ovulation in ewes by exogenous progestagen. Theriogenology, v.50, p.395-416, 1998.

OLIVEIRA, A.F.; NASCIMENTO, A.V.; TORRES, A.A.C. et al. Dinâmica folicular na sincronização de ovulação associada à administração de FSH-p em vacas da raça Nelore. Acta Sci. Vet., v.35, supl. 3, p.s1119, 2007.

PROSPERI, C.P.; TORRES, C.A.A.; MAFFILI, V.V. Indução de estro em cabras Saanen nulíparas utilizando-se diferentes tempos de exposição ao progestágeno. Rev. Bras. Zootec., v.27, p.481-483, 2003.

ROY, F.; MAUREL, M. C.; COMBES, B. The negative effect of repeated equine chorionic gonadotropin treatment on subsequent fertility in Alpine goats is due to a humoral immune response involving the major histocompatibility complex. Biol. Reprod., v.60, p.805-813, 1999.

RUBIANES, E.; DE CASTRO, T.; CARBAJAL, B. Effect of high progesterone levels during the growing phase of the dominant follicle of wave 1 in ultrasonically monitored ewes. Can. J. Anim. Sci., v.76, p.473-475, 1996.

SQUIRES, E.L. Perspectivas para o uso de biotecnologias na reprodução equina. Acta Sci. Vet., v.33, Supl. 1, p.69-82, 2005. 